\title{
Disease-dependent Differently Methylated Regions (D-DMRs) of DNA are Enriched on the $X$ Chromosome in Uterine Leiomyoma
}

\author{
Ryo MAEKAWA ${ }^{1)}$, Shintaro YAGI ${ }^{2)}$, Jun OHGANE²), Yoshiaki YAMAGATA ${ }^{1)}$, Hiromi ASADA ${ }^{1)}$, \\ Isao TAMURA ${ }^{1)}$, Norihiro SUGINO ${ }^{1)}$ and Kunio SHIOTA ${ }^{2)}$ \\ ${ }^{1)}$ Department of Obstetrics and Gynecology, Yamaguchi University Graduate School of Medicine, Ube 755-8505 and \\ ${ }^{2)}$ Laboratory of Cellular Biochemistry, Department of Animal Resource Sciences/Veterinary Medical Sciences, The \\ University of Tokyo, Tokyo 113-8657, Japan
}

\begin{abstract}
Uterine leiomyoma is the most common benign tumor in women. Although responsible gene mutations have not been found in leiomyomas, they represent a progressive disease with irreversible symptoms. To characterize epigenetic features of uterine leiomyomas, the DNA methylation status of a paired sample of leiomyoma and normal myometrium was subjected to a microarray-based DNA methylation analysis with restriction tag-mediated amplification (D-REAM). In the leiomyoma, we identified an aberrant DNA methylation status for 463 hypomethylated and 318 hypermethylated genes. Although these changes occurred on all chromosomes, aberrantly hypomethylated genes were preferentially located on the $\mathrm{X}$ chromosome. Using paired samples of normal myometrium and leiomyoma from 6 hysterectomy patients, methylation-sensitive quantitative real-time PCR revealed 14 shared X chromosome genes with an abnormal DNA hypomethylation status (FAM9A, CPXCR1, CXORF45, TAF1, NXF5, VBP1, GABRE, DDX53, FHL1, BRCC3, DMD, GJB1, AP1S2 and PCDH11X) and one hypermethylated locus (HDAC8). Expression of $\mathrm{XIST}$, which is involved in $\mathrm{X}$ chromosome inactivation, was equivalent in the normal myometrium and leiomyoma, indicating that the epigenetic abnormality on the $\mathrm{X}$ chromosome did not result from aberration of XIST gene expression. Based on these data, a unique epigenetic signature for uterine leiomyomas has emerged. The 14 hypomethylated and one hypermethylated loci provide valuable biomarkers for understanding the molecular pathogenesis of leiomyoma.

Key words: Disease-dependent differently methylated regions (D-DMRs), DNA methylation, Epigenetics, Leiomyoma, $\mathrm{X}$ chromosome
\end{abstract}

(J. Reprod. Dev. 57: 604-612, 2011)

$\mathbf{U}$ terine leiomyomas are the most common uterine tumors in reproductive-age women with a prevalence of $20-25 \%$ [1]. Leiomyomas frequently cause serious gynecological problems such as pelvic pain, menorrhagia, dysmenorrhea, infertility and recurrent pregnancy loss $[2,3]$. In addition, uterine leiomyomas are the most common indication for hysterectomy.

Despite their high prevalence rate and distressing effect on women's reproduction, the pathogenesis of uterine leiomyomas remains unclear. Factors such as African descent, high body mass index, meat consumption, early menarche, hypertension and a history of pelvic inflammatory disease place women at greater risk for uterine leiomyoma. In contrast, women using hormonal contraception, who smoke, are parous and who consume green vegetables have lower risk [4-6]. These findings suggest that uterine leiomyomas develop not only by inherited genomic abnormalities but also by unfavorable environmental exposures.

DNA methylation is one of the most well-characterized epigenetic marks. It is involved in gene-silencing, gene-stability and DNA duplication [7-9]. DNA methylation profiles define and distinguish between each type of normal cell $[10,11]$ and have been

Received: March 11, 2011

Accepted: May 17, 2011

Published online in J-STAGE: June 17, 2011

(C)2011 by the Society for Reproduction and Development

Correspondence: K Shiota (e-mail: ashiota@mail.ecc.u-tokyo.ac.jp) and

N Sugino (e-mail: sugino@yamaguchi-u.ac.jp) used to characterize abnormal cells [8]. For each cell type, establishing and maintaining the specific DNA methylation profile of the cells is necessary for cellular integrity, and disruption of the profile may have serious health consequences. We previously reported a microarray-based DNA methylation analysis, named tissue-dependent differentially methylated regions (T-DMR) profiling with restriction tag-mediated amplification (D-REAM), which identifies genes with differentially methylated restriction enzyme sites [12]. The DNA methylation profiles that encompass hypo- and hypermethylated T-DMRs are unique to a given cell type and providing a new paradigm for defining normal cell lineages $[13,14]$.

Irreversible changes to cell phenotypes may arise from epimutations as well as from mutations. Epimutations are defined as heritable changes of gene function without mutation of the gene. In addition to mutations, epimutations of tumor suppressor genes have been shown to participate in carcinogenesis [15]. Benign tumors and chronic diseases are also associated with epimutations of restricted genes [16]. Using the method of Restriction Landmark Genomic Scanning, we previously showed that uterine leiomyomas had aberrant DNA methylation compared with a matched patient's normal myometrium [17]. Global hypomethylation and imbalanced expression of DNA methyltransferases were also found in uterine leiomyoma $[17,18]$. The question "Are there diseasedependent differently methylated regions (D-DMRs) in uterine leiomyomas?" awaits an answer. 
Table 1. Specimens of leiomyomas and corresponding myometriums from 6 cases

\begin{tabular}{|c|c|c|c|c|c|c|}
\hline & Age (y.o.) & Menstrual cycle & Day of menstrual cycle & Menstrual phase & Type & Diameter (mm) \\
\hline Case 1 & 43 & $25-28$ & 13 & Follicular & Intramural & 90 \\
\hline Case 2 & 39 & unknown & 10 & Follicular & Intramural & 70 \\
\hline Case 3 & 49 & 25 & 16 & Unknown & Intramural & 160 \\
\hline Case 4 & 40 & 30 & 5 & Follicular & Intramural & 85 \\
\hline Case 5 & 38 & $27-30$ & 20 & Luteal & Intramural & 120 \\
\hline Case 6 & 35 & 28 & 16 & Luteal & Submucosal & 80 \\
\hline
\end{tabular}

D-REAM was performed for case 6 , and all samples including case 6 were analyzed using methylation-sensitive quantitative real-time PCR Phase: menstrual phases when specimens were obtained.

Here, we have analyzed the genome-wide DNA methylation status of leiomyoma and matched myometrium by D-REAM. We identified a number of D-DMRs in human uterine leiomyomas that provide epigenetic biomarkers to better understand the molecular pathogenesis of this disease.

\section{Materials and Methods}

\section{Tissue preparations}

Paired specimens of uterine leiomyoma and corresponding normal myometrium were obtained from 6 women who had a single leiomyoma nodule and underwent hysterectomy (Table 1). None of the women had received previous treatment with sex steroid hormones or gonadotropin releasing hormone $(\mathrm{GnRH})$ analogs. Specimens were dissected immediately from the full thickness uterine wall without the endometrium and serosa after removal of the uterus, immersed in liquid nitrogen and stored at $-80 \mathrm{C}$ until DNA/ RNA extraction. Informed consent was obtained from all participating patients, and ethical approval was obtained from Yamaguchi University Graduate School of Medicine and Graduate School of Agricultural and Life Sciences of the University of Tokyo.

\section{$D-R E A M$ analysis}

The D-REAM method was adapted from our previous study [12]. Briefly, $250 \mathrm{ng}$ of genomic DNA digested with HpyCH4IV (New England BioLabs, Ipswich, MA, USA), a methylation-sensitive restriction enzyme, was amplified by ligation-mediated PCR with the adaptors and PCR primers listed in Supplementary Table $\mathrm{S} 1$. Ten $\mu \mathrm{g}$ of amplified DNA was used for hybridization with Human Promoter 1.0R Tiling Array (Affymetrix, Santa Clara, CA, USA), and the data were processed by Model-based Analysis of Tiling-arrays (MAT) [19], with the probe library file (.bpmap file) for hg18 assembled human genome obtained from the MAT website (http://liulab.dfci.harvard.edu/MAT). In this study, we defined the differentially methylated HpyCH4IV sites between normal myometrium and leiomyomas as D-DMRtags. The lists of DDMRtags for each comparison were obtained by MAT with a pvalue of $10^{-7}$ as the cutoff value. When there were hypomethylated and hypermethylated D-DMRtags in one gene loci, the gene was counted as hypomethylated and hypermethylated, respectively. When one D-DMRtag was located near the promoter regions of two or more genes, the genes were recognized as genes with individual D-DMRtags. The NCBI Reference Sequence (RefSeq) database was used as a reference gene in this study. The data were visualized using the Integrated Genome Browser (IGB, http:// www.affymetrix.com/support/-developer/tools/download_igb.affx). D-REAM was performed between normal myometrium tissue and leiomyoma tissue taken from one patient, which enabled us to exclude the genomic influence such as SNPs. Gene mutation, if it occurs in uterine leiomyomas, may affect the exact estimation of the DNA methylation profiles in D-REAM analysis.

\section{Bisulfite restriction mapping and sequencing}

Bisulfite reactions were carried out as previously described [20]. The bisulfite-converted DNA was amplified by PCR with the primers listed in Supplementary Table S2. The thermocycling program was an initial cycle of $94 \mathrm{C}$ for $10 \mathrm{~min}$, then 43 cycles of $94 \mathrm{C}$ for $30 \mathrm{sec}, 60 \mathrm{C}$ for $30 \mathrm{sec}$ and $72 \mathrm{C}$ for $1 \mathrm{~min}$ and finally $10 \mathrm{~min}$ of final extension at $72 \mathrm{C}$. For restriction mapping, 1/2 each of the PCR products was treated with or without HpyCH4IV, and the resulting DNA fragments were assessed with a microchip electrophoresis system (MultiNA, Shimadzu Biotech, Kyoto, Japan). For bisulfite sequencing, the PCR products were subcloned into pGEM-T easy vector (Promega, Tokyo, Japan), and 10 or more clones were sequenced using an ABI 3130 sequencer with a BigDye terminator kit (Applied Biosystems, Foster City, CA, USA).

\section{Methylation-sensitive quantitative real-time PCR}

Methylation-sensitive quantitative real-time PCR was performed as previously described [21]. This method utilizes digestion of genomic DNA with HpyCH4IV prior to quantitative PCR. Ten ng of the DNA treated with or without HpyCH4IV were subjected to PCR with the primer pair amplifying the genomic fragment containing the HpyCH4IV site. The amplification was monitored by SYBR green in Power SYBR green PCR Master Mix (Applied Biosystems) using an ABI Prism 7500 Real-Time PCR System (Applied Biosystems) according to the manufacturer's protocols. The DNA methylation level at each HpyCH4IV site was determined as the proportion of the amount of undigested DNA in the HpyCH4IV-treated solution to that without the HpyCH4IV treatment. To obtain the proportion of the total amount of HpyCH4IV-treated DNA to untreated DNA, real-time PCR was performed using primer pairs of SALL1 and GATA1 that were designed to amplify fragments without the HpyCH4IV site. The primers used in the PCRs are listed in Supplementary Table S3.

\section{$R T-P C R$}

Total RNAs were isolated from tissues using TRIzol reagent 
A

Tissue A

Methylated

HpyCH4IV site

CH4IV site

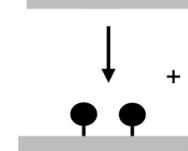

HpyCH4IV cut

+ R-adaptor pair ligation

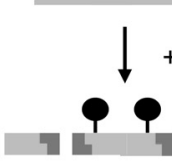

Taq I cut

오오

$+\mathrm{N}$-adaptor pair ligation

$\mathrm{PCR}_{\rightarrow} \stackrel{\mathrm{B}}{\rightarrow}$

우

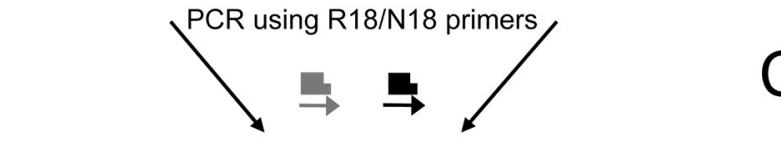

B

C
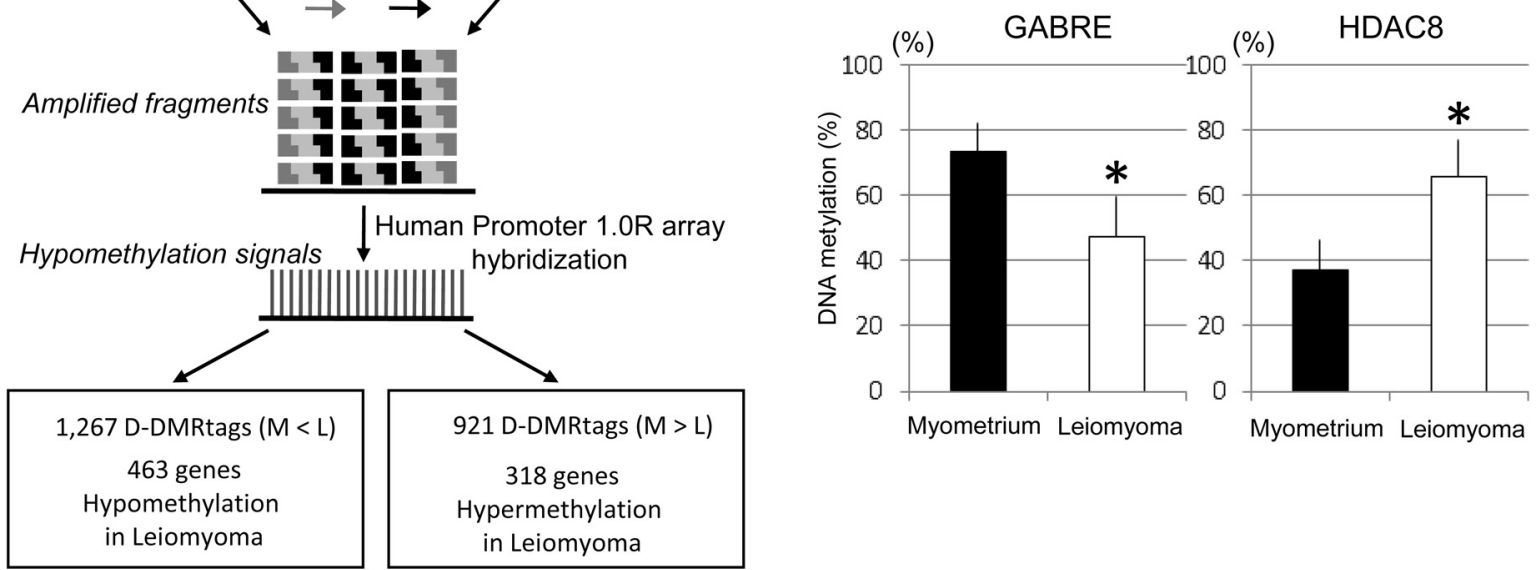

Fig. 1. Genome-wide DNA methylation analysis by D-REAM and validation of typical potential D-DMRs. A: Illustration of the D-REAM method. Genomic DNA was digested with HpyCH4IV and TaqI, followed by adaptor ligation. PCR reaction amplifies unmethylated HpyCH4IV-TaqI and HpyCH4IV-HpyCH4IV fragments selectively. Amplified fragments were hybridized with human promoter array. Array signal intensities were analyzed to identify regions corresponding to fragments in unmethylated HpyCH4IV loci. Comparison of signals between two different samples enables identification of differentially methylated regions. A total of 2,188 D-DMRtags were identified by comparison of a matched pair of leiomyoma and myometrium. There were 1,267 leiomyoma-hypomethylated tags corresponding to 463 genes, while there were 921 leiomyoma-hypermethylated tags corresponding to 318 genes. B: Positions of potential D-DMRs and D-DMRtags in the promoter regions of GABRE and HDAC8. The upper section displays the positions of HpyCH4IV. Boxes and arrowheads represent potential D-DMRs and D-DMRtags, respectively. The middle section shows integrated genome browser (Affymetrix) plots of comparative microarray signals corresponding to the potential D-DMRs from $7500 \mathrm{bp}$ upstream to $2500 \mathrm{bp}$ downstream of the TSS. C: The methylation levels (\%) at the potential DDMRtags of GABRE and HDAC8. One sample (Case 6) was analyzed three times by sodium bisulfite restriction mapping. Values are means \pm SEM of three different assays. $* \mathrm{P}<0.01 v s$. myometrium (Student's $t$-test).

(Invitrogen, Carlsbad, CA, USA) and reverse-transcribed a using SuperScript III First-Strand Synthesis System (Invitrogen) according to the manufacturer's protocol. The synthesized DNA was subjected to PCR reactions with the primer sets listed in Supplementary Table S4. Primers for $\beta$-actin were used as an internal control for the quantity of the RNA used. PCR was performed using an iCycler (Bio-Rad Laboratories). The thermocycling program was an initial cycle of $94 \mathrm{C}$ for $10 \mathrm{~min}$, then 22-35 cycles of $94 \mathrm{C}$ for $30 \mathrm{sec}, 55-60 \mathrm{C}$ for $30 \mathrm{sec}$ and $72 \mathrm{C}$ for $1 \mathrm{~min}$ and finally final extension for $10 \mathrm{~min}$ at $72 \mathrm{C}$. The resulting products were subjected to agarose gel electrophoresis.

\section{Bioinformatics}

Chromosome distribution analysis was performed using DAVID 2007 (http://niaid.abcc.ncifcrf.gov/) [22]. MultiExperiment Viewer (MeV in the TM4 microarray Software Suite; http:// www.tm4.org/mev.html) was used for heat map generation [23]. QUMA (http://quma.cdb.riken.jp/) was used to analyze the bisulfite sequencing data [24]. Transcriptome microarray data were obtained from the National Center for Biotechnology Information (NCBI) Gene Expression Omnibus (GEO) database (ID: GSE593, http://www.ncbi.nlm.nih.gov/geo/query/acc.cgi?acc= GSE593) [25]. 
Table 2. Chromosomal distributions of the hypomethylated and hypermethylated genes in uterine leiomyomas

\begin{tabular}{|c|c|c|c|c|c|}
\hline \multicolumn{3}{|c|}{ Hypomethylated genes } & \multicolumn{3}{|c|}{ Hypermethylated genes } \\
\hline Chromosome & Genes & $P$ value & Chromosome & Genes & $P$ value \\
\hline $\mathrm{X}$ & 45 & $2.52 \mathrm{E}-08$ & 6 & 39 & $1.26 \mathrm{E}-06$ \\
\hline 11 & 43 & 0.002288982 & 18 & 12 & 0.006384167 \\
\hline 5 & 30 & 0.038070553 & 13 & 12 & 0.018669816 \\
\hline 20 & 19 & 0.102006284 & 1 & 45 & 0.023883559 \\
\hline 7 & 28 & 0.154158271 & 7 & 24 & 0.025210846 \\
\hline 14 & 19 & 0.187474909 & 2 & 25 & 0.197124289 \\
\hline 12 & 29 & 0.249094781 & 3 & 21 & 0.279695924 \\
\hline 4 & 21 & 0.325571198 & 4 & 14 & 0.430285933 \\
\hline 2 & 32 & 0.399392564 & 10 & 13 & 0.551204625 \\
\hline 1 & 50 & 0.462453947 & 8 & 11 & 0.631252799 \\
\hline 8 & 17 & 0.512311867 & 5 & 14 & 0.63561596 \\
\hline 15 & 14 & 0.686208806 & 20 & 8 & 0.779883544 \\
\hline 17 & 25 & 0.768293785 & 12 & 13 & 0.905374017 \\
\hline 21 & 5 & 0.852744569 & 16 & 10 & 0.907434784 \\
\hline 18 & 4 & 0.965874601 & 17 & 13 & 0.961330622 \\
\hline 10 & 11 & 0.985370937 & 11 & 13 & 0.984311775 \\
\hline 19 & 22 & 0.990441405 & 15 & 5 & 0.989164547 \\
\hline 6 & 15 & 0.99210258 & 9 & 7 & 0.990091868 \\
\hline 9 & 11 & 0.992668284 & 22 & 3 & 0.995213304 \\
\hline 13 & 3 & 0.996932445 & $\mathrm{X}$ & 2 & 0.999030061 \\
\hline 22 & 4 & 0.998837234 & 14 & 3 & 0.99945488 \\
\hline 16 & 9 & 0.998977446 & 19 & 2 & 1 \\
\hline 3 & 10 & 0.999966169 & 21 & 1 & 1 \\
\hline
\end{tabular}

Chromosomal distribution was analyzed using DAVID 2007 [22], and P-values were calculated by Fisher's exact test in DAVID 2007.

\section{Results}

Genome-wide DNA methylation profile of uterine leiomyoma

D-REAM analysis (Fig.1A) detected 1,267 hypomethylated and 921 hypermethylated potential D-DMRtags in a leiomyoma (Case 6 in Table 1). These represented 463 and 318 gene loci, respectively (Fig.1A). Ten genes possessed both hypomethylated and hypermethylated potential D-DMRtags. Importantly, the potential D-DMRs of the leiomyomas were distributed across all chromosomes (Table 2), suggesting a genome-wide epigenetic abnormality. These data support our previous work that concluded that genome-wide alteration of DNA methylation status at potential D-DMRs underlies the pathogenesis of uterine leiomyoma [17].

To illustrate the D-REAM data, two detected gene tags are presented as examples. D-REAM detected the hypomethylated potential D-DMRtags upstream as well as downstream of the transcription start site of the GABRE gene and hypermethylated potential D-DMRtags upstream of the HDAC8 gene (Fig. 1B). The DNA methylation status at the potential D-DMRtags of these two genes was confirmed by bisulfite restriction mapping (Fig. 1C).

Figure 2 shows the distributions of potential D-DMRtags at the promoter regions. Hypermethylated genes tended to have fewer DDMRtags from -500 to +1000 bp compared with the other promoter region, which was different from the distribution of the hypomethylated genes.

Intriguingly, 45 genes out of 463 hypomethylated genes were located on the $\mathrm{X}$ chromosome, while only two hypermethylated genes (HDAC8, GPC3) were identified on the $\mathrm{X}$ chromosome. The 45 hypomethylated genes were distributed throughout the $\mathrm{X}$ chromosome. We therefore selected 30 potential D-DMRs from the 45 hypomethylated and 2 hypermethylated genes on the X chromosome and compared them in the normal myometriums and leiomyomas from 6 patients to distinguish patient-independent from patient-dependent variation (Fig. 3).

\section{Leiomyoma-hypomethylated D-DMRs on the X chromosome}

Methylation-sensitive quantitative real-time PCR confirmed that the potential D-DMRs of the 30 genes were differentially methylated between the normal myometrium and leiomyoma and found that DNA methylation levels varied among these genes (Fig. 3C). The HpyCH4IV sites of MID1, AP1S2, ACOT9, MSN, CPXCR1, $C X O R F 45$ and $P A S D 1$ were fully methylated in the normal myometrium, while in the leiomyoma, they exhibited 40-60\% methylation (MID1, AP1S2, ACOT9, MSN and CPXCR1) or 75$80 \%$ methylation (CXORF 45 and $P A S D$; Fig. 3C). Similarly, the methylation levels of TAF1 (75\%), PCDH11X (75\%), NXF5 (50\%), FHL1 (40\%) and MTM1 (50\%) in the normal myometrium were reduced to $20,50,10,20$ and $30 \%$, respectively, in the leiomyoma. HDAC8 showed $50 \%$ methylation in the normal myometrium but $80 \%$ methylation in the leiomyoma. We do not have direct evidence to show that $50 \%$ methylation is allele-dependent methylation at the DMRtag sites. We cannot exclude the 

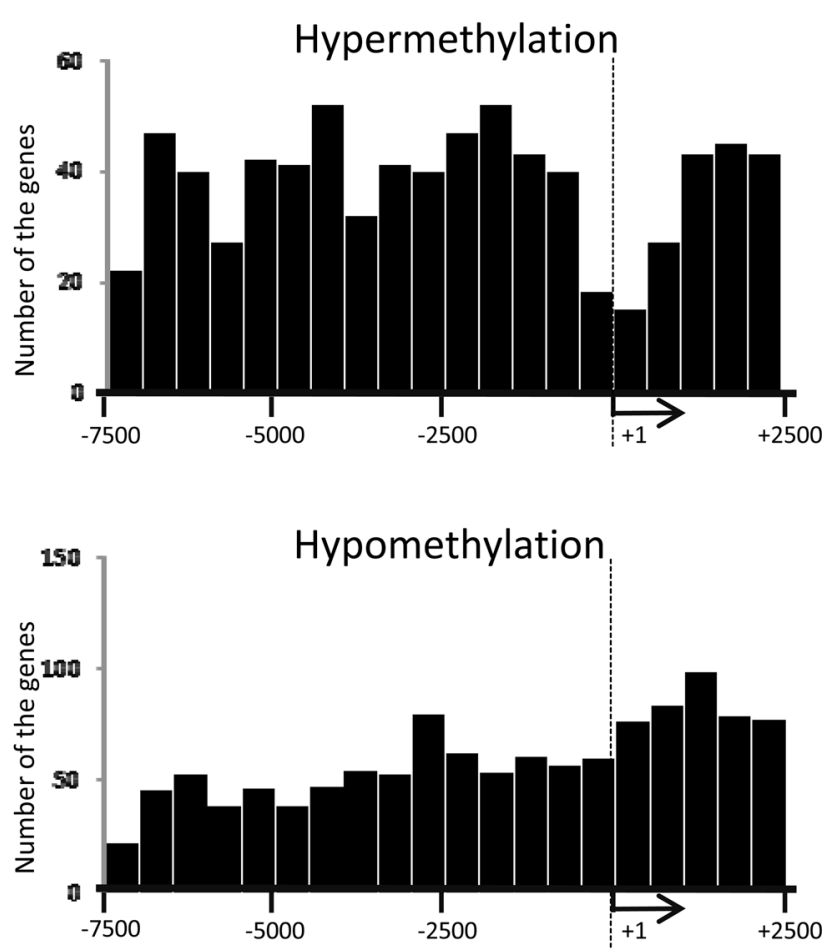

Fig. 2. The positions relative to the transcription start site (TSS) of the identified potential D-DMRtags in 318 aberrantly hypermethylated (upper) and 463 aberrantly hypomethylated (lower) genes. The y axis shows the number of the genes that possess D-DMRtags at the positions relative to the TSS indicated on the $\mathrm{x}$ axis.

possibility that $50 \%$ methylation may depend on the mixture of methylated cells and unmethylated cells. However, in the bisulfite sequencing analysis on several genes in Fig 5B, the methylated CpGs were distributed in an allele-independent manner. This result suggests an allele-independent methylation in each cell.

$X$ chromosome inactivation is unaffected in uterine leiomyoma

The promoter and the first exon of XIST should be 50\% methylated if $\mathrm{X}$ chromosome inactivation is normal [26]. The XIST promoter region showed about $50 \%$ methylation in the leiomyoma as well as in the normal myometrium in Case 6 (Fig. 4A). The expression of XIST was detected in all patients, although the expression was slightly higher in leiomyoma Case 1 (Fig. 4B). Expression of TSIX was not detected in both the leiomyoma and normal myometrium from any of the 6 patients (Fig. 4B).

The promoter region of $F M R 1$ and the first exon of $A R$ should be $50 \%$ methylated if $X$ chromosome inactivation is normal $[27,28]$. Indeed, in Case 6, FMRI and $A R$ were about $50 \%$ methylated in the normal myometrium exhibiting the typical pattern of genes regulated by $\mathrm{X}$ inactivation. In contrast, the Case 6 leiomyoma DNA methylation levels of these two promoters were reduced to less than $10 \%$ (Fig. 4A). These data suggest that in leiomyoma growths, there are genes with potential D-DMRs on the X chromosome that escape $\mathrm{X}$ inactivation.
DNA methylation profiles of the X chromosome as epigenetic biomarkers for leiomyoma

To investigate epigenetic biomarkers for uterine leiomyomas, we examined the methylation status of the 30 genes in five additional paired samples (Cases 1-5 in Table 1) together with Case 6 by methylation-sensitive quantitative real-time PCR (Fig. 5A). Among 29 hypomethylated genes, 14 genes shared the hypomethylated status in four or more of the six leiomyoma cases (Fig. 5A). In addition, $H D A C 8$ was hypermethylated in four of the six cases (Fig. 5A). These genes are possible D-DMRs, which can be differentiated from "potential D-DMR". The remaining 15 genes, which were hypomethylated in Case 6, had variable methylation states between the cases (Fig. 5A). Information on the possible DDMRtags including the HpyCH4IV sites is shown in Supplementary figure 1 .

Three genes that shared methylation abnormalities in leiomyomas were further analyzed for DNA methylation status by bisulfite sequencing (Fig. 5B). HpyCH4IV sites at -3013 and an additional CpG site ( -3033 bp) were hypomethylated in GABRE, while only the restriction site $(-48 \mathrm{bp})$ was hypomethylated in $P C D H 11 X$. In $H D A C 8$, hypermethylation was observed at an $H p y C H 4 I V$ site ($3533 \mathrm{bp})$ as well as at an additional $\mathrm{CpG}$ (-3536 bp).

\section{Discussion}

The present study shows that uterine leiomyomas are associated with genome-wide alterations in DNA methylation at multiple gene promoter regions. We focused on the $\mathrm{X}$ chromosome as a characteristic site of aberrant DNA methylation status in surgically removed uterine leiomyomas. From comparisons between diseased and normal tissues from the same uteri of 6 patients, we identified 14 hypomethylated genes and one hypermethylated gene located on the $\mathrm{X}$ chromosome that provide the first epigenetic signature for leiomyoma. These data provide novel clues for understanding the development and molecular pathogenesis of leiomyoma.

One of these clues is involvement of the $\mathrm{X}$ chromosome. Our data may be supported by a previous report that identified differences in the gene expression pattern between leiomyoma and the normal myometrium [29]. When we re-analyzed the raw array data in the GEO database [25], 15 genes of 463 hypomethylated genes had increased expression in leiomyomas, and 4 of the 15 genes were found to be located on the $\mathrm{X}$ chromosome. This finding predicted a hypomethylation status to account for the increased gene expression from the $\mathrm{X}$ chromosome. Skubitz et al. also reported that 4 genes, three of which are on the $\mathrm{X}$ chromosome, were especially overexpressed in leiomyomas compared with the normal myometrium [30]. Combined with our findings, these data suggest that aberrant DNA methylation leads to aberrant gene expression.

RNA accumulation of XIST throughout the $\mathrm{X}$ chromosome is a trigger for $\mathrm{X}$ chromosome inactivation, followed by histone modification by polycomb repressive complex [31]. It is unlikely that an aberration of the XIST gene is involved in uterine leiomyomas because there was no difference in methylation status of the XIST gene promoter between leiomyomas and the normal myometrium and XIST mRNA expression was not inhibited in uterine leiomyo- 
A
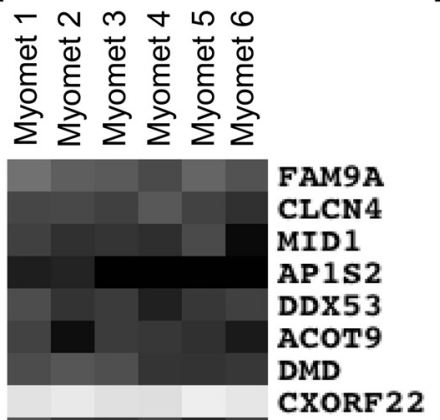

SSX6

NUDT 11

MSN

GJB 1

TAF 1

HDAC8

RNF 12

CPXCR 1

TGIF 2LX

PCDH $11 \mathrm{X}$

NXF5

PRPS1

CXORF 45

MBNL 3

HS6ST2

FHL 1

SPANXE

MTM1

PASD 1

GABRE

VBP 1

BRCC3

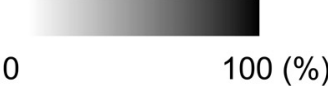

B

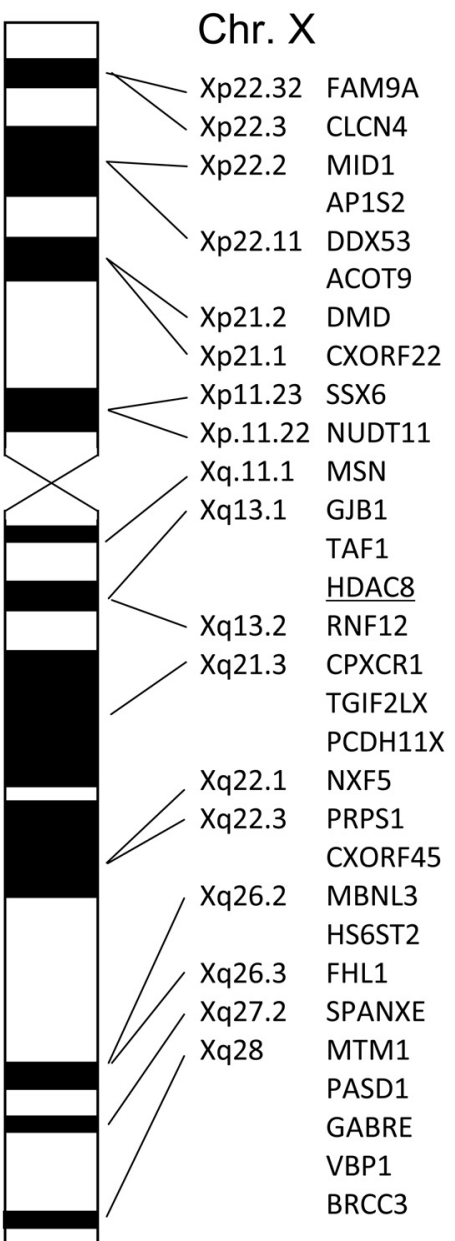

C

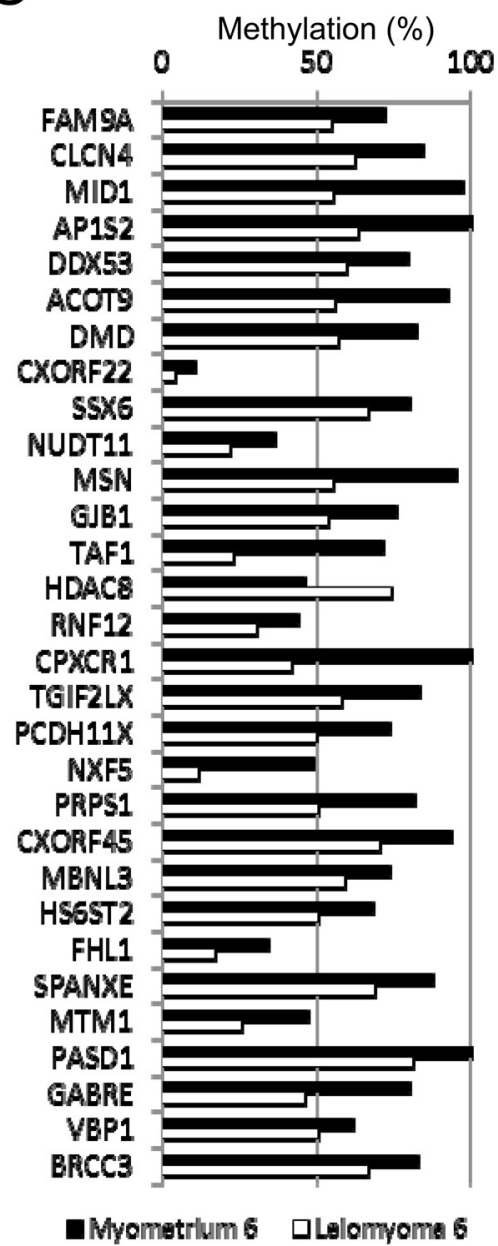

Fig. 3. The DNA methylation patterns of potential D-DMR genes on the X chromosome. A: The methylation levels (\%) of the potential D-DMR genes in six normal myometriums analyzed by MS-PCR. The result shows almost identical DNA methylation patterns. B: The locations of 29 hypomethylated genes and one hypermethylated gene (underlined) in leiomyomas are shown in the $\mathrm{X}$ chromosome. These 29 hypomethylated genes were distributed throughout the X chromosome. C: Methylation levels of 29 hypomethylated genes and one hypermethylated gene were analyzed at potential D-DMR sites by methylation-sensitive quantitative real-time PCR.

mas. However, since the 45 hypomethylated genes were distributed throughout the $\mathrm{X}$ chromosome and the methylation status of the FMR1 and $A R$ genes was also hypomethylated in uterine leiomyomas, we cannot exclude a possibility that X-inactivation machineries "except XIST" could be disturbed in leiomyomas. It is also important to note that many of the D-DMRs were found on the autosomes in addition to the $\mathrm{X}$ chromosome. T-DMRs have been identified on normal sex chromosomes and autosomes [12-15, 32]. Local regulation of the DNA methylation status of T-DMRs may depend on the combination of global regulators and other undefined factors involved in the histone modification system [33]. Therefore, the D-DMRs found in uterine leiomyomas may now be thought to arise due to global disruption or globally inappropriate maintenance of T-DMRs.
Alterations in regional methylation patterns have been reported to be involved in pathogenesis of malignant tumors [34-36]. In particular, hypermethylation of the promoter region is associated with silencing of tumor suppressor/DNA repair genes, transcriptional activation of oncogenes and loss of imprinting in malignant cells [34-36]. It is well known that the increased incidence of DNA hypermethylation in the $\mathrm{CpG}$ island in the proximal promoter or the first exon of estrogen receptor (ER)-alpha is highly associated with the loss of ER-alpha expression in breast cancers [37, 38]. On the other hand, our result showed low incidence of DNA hypermethylation in the promoter region around the transcription start site. We also reported that the DNA hypomethylation, but not hypermethylation, was observed in the distal promoter region of ER-alpha outside the $\mathrm{CpG}$ island in uterine leiomyomas [20]. 
A

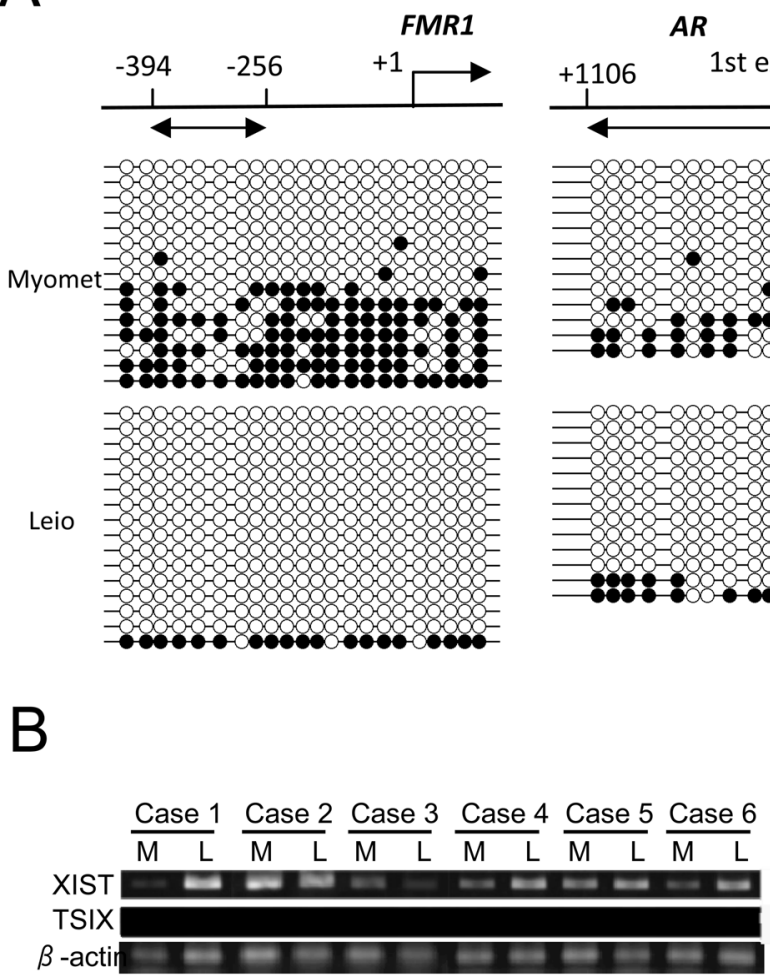

Fig. 4. DNA methylation status of genes exhibiting the typical pattern of $\mathrm{X}$ inactivation in the normal myometrium and the XIST mRNA expression in paired samples of leiomyoma and normal myometrium. A: DNA methylation status of the FMR1 promoter region, the first exon of the $A R$ gene and the promoter/first-exon region of XIST in Case 6 were analyzed by sodium bisulfite sequencing. Open and filled circles indicate unmethylated and methylated CpGs, respectively. B: XIST mRNA expressions in paired samples of leiomyoma and normal myometrium were examined for six cases. M, normal myometrium; $\mathrm{L}$, leiomyoma.

These observations suggest that epigenetic aberration in uterine leiomyomas, benign tumors, may be different from that in malignant tumors. Furthermore, it is unclear why there is a difference in the distribution of hypermethylated and hypomethylated tags in the promoter region in uterine leiomyomas. Further studies are needed to investigate whether low incidence of genes with hypermethylated tags in the proximal promoter region is pathophysiologically significant in uterine leiomyomas.

Among our 6 cases, fifteen genes varied in methylation status. Heterogeneity of DNA methylation was also observed in our previous report [17]. In several cancers, the methylation status of specific genes correlates with disease progression [39-42]. The variable methylation profile of the genes we studied may reflect an intermediate state of progression and prognosis of leiomyoma. Further studies will be needed to clarify the relationship between DNA methylation status and clinical characteristics such as tumor size, tumor growth, and sensitivity or resistance to medication.

This is the first report to identify the common genes with aberrant DNA methylation on the $\mathrm{X}$ chromosome in uterine leiomyomas. We propose that the hypomethylated D-DMRs of FAM9A, CPXCR1, CXORF45, TAF1, NXF5, VBP1, GABRE,
DDX53, FHL1, BRCC3, DMD, GJB1, AP1S2 and PCDH11X and the hypermethylated D-DMR of $H D A C 8$ will be valuable for better understanding the molecular mechanisms and biology of uterine leiomyoma. In addition, the aberrantly methylated gene profile observed in uterine leiomyomas may become a useful epigenetic biomarker for uterine leiomyomas, especially in the case that the aberrant methylation of those genes does not reflect gene expression. These aspects may also lead to new strategies for prevention or prediction of prognosis for uterine leiomyoma patients. Furthermore, the present study including D-REAM may help to specify molecules responsible for global DNA demethylation that is frequently seen not only in uterine leiomyomas but also in most cancers.

\section{Acknowledgments}

We thank Dr A Croy (Queen's University, ON, Canada) for critically reviewing the manuscript. This work was supported by grants from the National Institute of Biomedical Innovation (NIBIO); New Energy and Industrial Technology Development Organization (NEDO); Grants-in-Aid for Scientific Research 
A

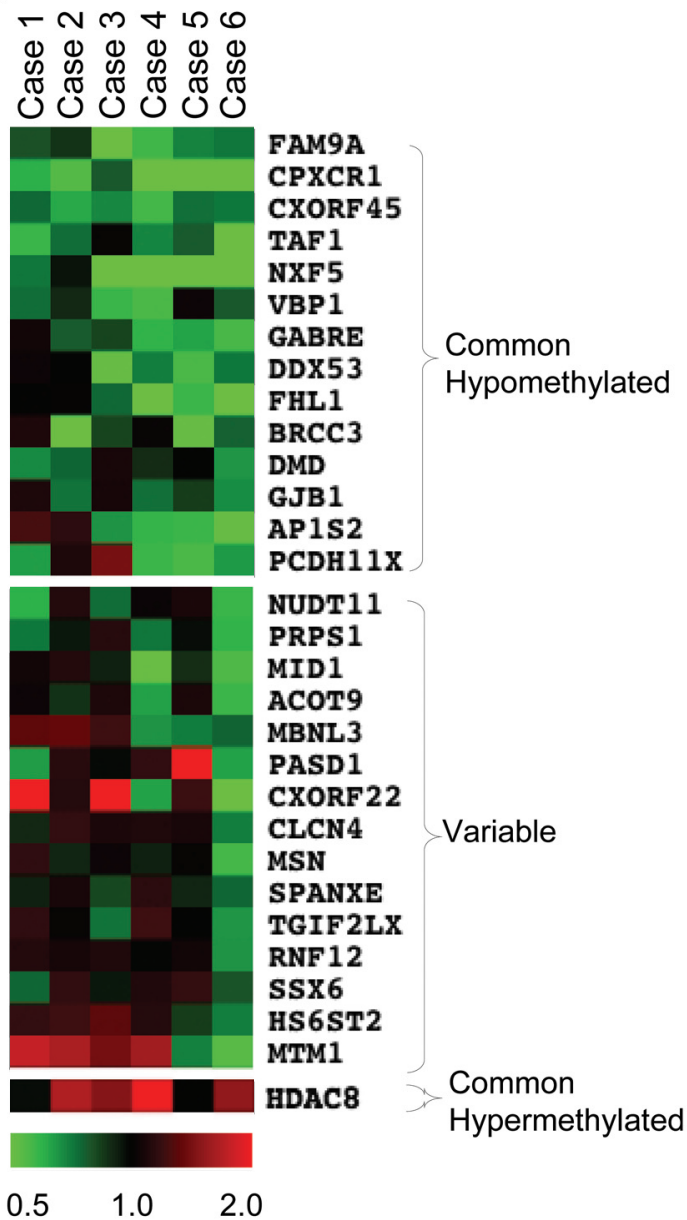

B
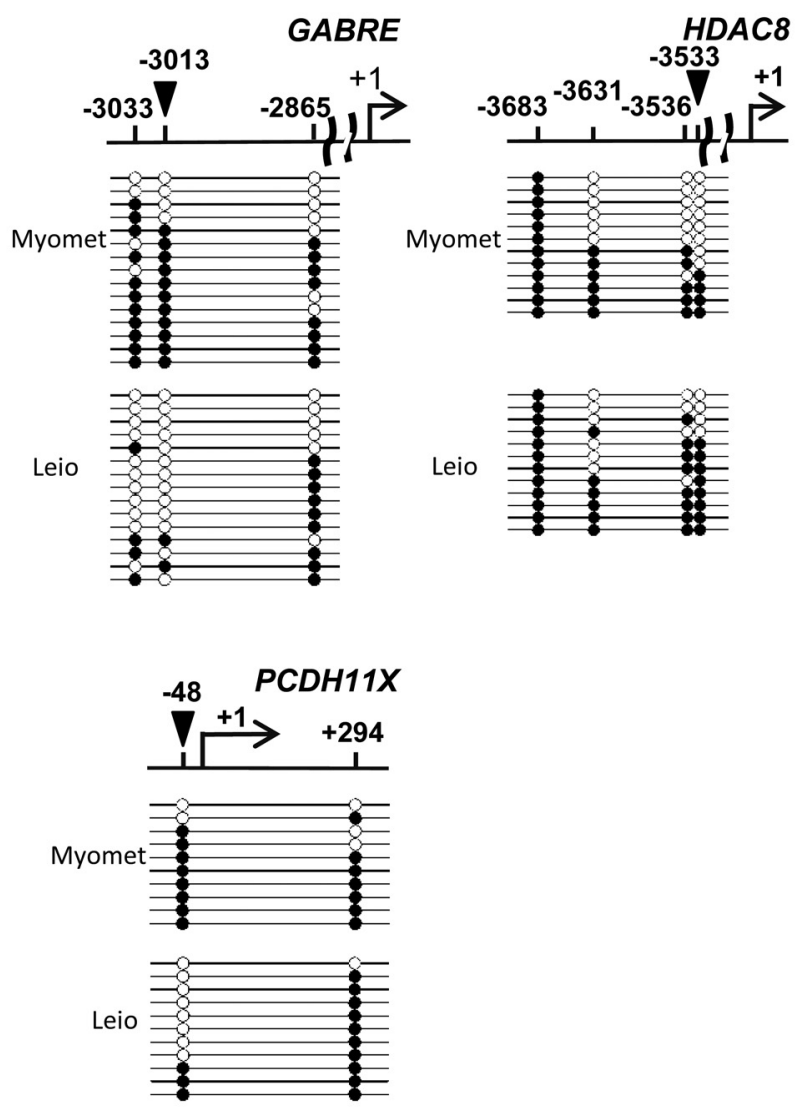

Fig. 5. DNA methylation patterns of shared and variable D-DMRs and DNA methylation status of shared D-DMRs analyzed by sodium bisulfite sequencing. A: The relative methylation levels of leiomyoma tissue to normal myometrium tissue are shown. Among 31 hypomethylated genes we examined, 14 genes showed similar hypomethylation in four or more of the six cases. HDAC8 exhibited a shared hypermethylation profile in four of the six cases. The remaining 15 genes showed variable methylation patterns. The ranges of methylation level ratios are shown at the bottom of the panels. Green and red coloring denote aberrant hypomethylation and aberrant hypermethylation, respectively, in a leiomyoma compared with a normal myometrium. Case 6 was analyzed by DREAM. B: Sodium bisulfite sequencing of the common D-DMR regions including D-DMRtags in GABRE, PCDH11X and $H D A C 8$. Open and closed circles represent unmethylated and methylated CpGs, respectively.

(KAKENHI) from the Ministry of Education, Culture, Sports, Science and Technology (MEXT), Japan, 20062003, 20591918, 21592099 and 21791559; and a Health Labour Sciences Research Grant from the Ministry of Health, Labour and Welfare (MHLW), Japan.

\section{References}

1. Vollenhoven BJ, Lawrence AS, Healy DL. Uterine fibroids: a clinical review. $\mathrm{Br} J$ Obstet Gynaecol 1990; 97: 285-298.

2. Stewart EA. Uterine fibroids. Lancet 2001; 357: 293-298

3. Bajekal N, Li TC. Fibroids, infertility and pregnancy wastage. Hum Reprod Update 2000; 6: 614-620

4. Chiaffarino F, Parazzini F, La Vecchia C, Chatenoud L, Di Cintio E, Marsico S. Diet and uterine myomas. Obstet Gynecol 1999; 94: 395-398.
5. Faerstein E, Szklo M, Rosenshein N. Risk factors for uterine leiomyoma: a practicebased case-control study. I. African-American heritage, reproductive history, body size, and smoking. Am J Epidemiol 2001; 153: 1-10.

6. Faerstein E, Szklo M, Rosenshein NB. Risk factors for uterine leiomyoma: a practicebased case-control study. II. Atherogenic risk factors and potential sources of uterine irritation. Am J Epidemiol 2001; 153: 11-19.

7. Li E. Chromatin modification and epigenetic reprogramming in mammalian development. Nat Rev Genet 2002; 3: 662-673.

8. Shiota K, Yanagimachi R. Epigenetics by DNA methylation for development of nor mal and cloned animals. Differentiation 2002; 69: 162-166.

9. Shiota K. DNA methylation profiles of $\mathrm{CPG}$ islands for cellular differentiation and development in mammals. Cytogenet Genome Res 2004; 105: 325-334.

10. Shiota K, Kogo Y, Ohgane J, Imamura T, Urano A, Nishino K, Tanaka S, Hattori N Epigenetic marks by DNA methylation specific to stem, germ and somatic cells in mice. Genes Cells 2002; 7: 961-969.

11. Lieb JD, Beck S, Bulyk ML, Farnham P, Hattori N, Henikoff S, Liu XS, Okumura K, Shiota K, Ushijima T, Greally JM. Applying whole-genome studies of epigenetic regulation to study human disease. Cytogenet Genome Res 2006; 114: 1-15. 
12. Yagi S, Hirabayashi K, Sato S, Li W, Takahashi Y, Hirakawa T, Wu G, Hattori N, Ohgane J, Tanaka S, Liu XS, Shiota K. DNA methylation profile of tissue-dependent and differentially methylated regions (T-DMRs) in mouse promoter regions demonstrating tissue-specific gene expression. Genome Res 2008; 18: 1969-1978.

13. Takasugi M, Yagi S, Hirabayashi K, Shiota K. DNA methylation status of nuclearencoded mitochondrial genes underlies the tissue-dependent mitochondrial functions. BMC Genomics 2010; 11: 481.

14. Sato S, Yagi S, Arai Y, Hirabayashi K, Hattori N, Iwatani M, Okita K, Ohgane J, Tanaka S, Wakayama T, Yamanaka S, Shiota K. Genome-wide DNA methylation profile of tissue-dependent and differentially methylated regions (T-DMRs) residing in mouse pluripotent stem cells. Genes Cells 2010; 15: 607-618.

15. Ushijima T, Okochi-Takada E. Aberrant methylations in cancer cells: where do they come from? Cancer Sci 2005; 96: 206-211.

16. Fujiki K, Kano F, Shiota K, Murata M. Expression of the peroxisome proliferator activated receptor gamma gene is repressed by DNA methylation in visceral adipose tissue of mouse models of diabetes. BMC Biol 2009; 7: 38 .

17. Yamagata Y, Maekawa R, Asada H, Taketani T, Tamura I, Tamura H, Ogane J, Hattori N, Shiota K, Sugino N. Aberrant DNA methylation status in human uterine leiomyoma. Mol Hum Reprod 2009; 15: 259-267.

18. Li S, Chiang T, Richard-Davis G, Barrett J, Mclachlan J. DNA hypomethylation and imbalanced expression of DNA methyltransferases (DNMT1, 3A, and 3B) in human uterine leiomyoma. Gynecol Oncol 2003; 90: 123-130.

19. Johnson WE, Li W, Meyer CA, Gottardo R, Carroll JS, Brown M, Liu XS. Modelbased analysis of tiling-arrays for ChIP-chip. Proc Natl Acad Sci USA 2006; 103: 1245712462 .

20. Asada H, Yamagata Y, Taketani T, Matsuoka A, Tamura H, Hattori N, Ohgane J, Shiota K, Sugino N. Potential link between estrogen receptor-alpha gene hypomethylation and uterine fibroid formation. Mol Hum Reprod 2008; 14: 539-545.

21. Hattori N, Abe T, Suzuki M, Matsuyama T, Yoshida S, Li E, Shiota K. Preference of DNA methyltransferases for $\mathrm{CpG}$ islands in mouse embryonic stem cells. Genome Res 2004; 14: 1733-1740.

22. Huang da W, Sherman BT, Tan Q, Kir J, Liu D, Bryant D, Guo Y, Stephens R, Baseler MW, Lane HC, Lempicki RA. DAVID Bioinformatics resources: expanded annotation database and novel algorithms to better extract biology from large gene lists. Nucleic Acids Res 2007; 35: W169-175.

23. Saeed AI, Sharov V, White J, Li J, Liang W, Bhagabati N, Braisted J, Klapa M, Currier T, Thiagarajan M, Stum A, Snuffin M, Rezantsev A, Popov D, Ryltsov A, Kostukovich E, Borisovsky I, Liu Z, Vinsavich A, Trush V, Quackenbush J. TM4: a free, open-source system for microarray data management and analysis. Biotechniques 2003; 34: 374-378.

24. Kumaki Y, Oda M, Okano M. QUMA: quantification tool for methylation analysis. Nucleic Acids Res 2008; 36: W170-175.

25. Barrett T, Troup DB, Wilhite SE, Ledoux P, Evangelista C, Kim IF, Tomashevsky M, Marshall KA, Phillippy KH, Sherman PM, Muertter RN, Edgar R. NCBI GEO: archive for functional genomics data sets--10 years on. Nucleic Acids Res 2011; 39: D1005-1010.

26. Norris D, Patel D, Kay G, Penny G, Brockdorff N, Sheardown S, Rastan S. Evidence that random and imprinted Xist expression is controlled by preemptive methylation.
Cell 1994; 77: 41-51.

27. Stoger R, Kajimura TM, Brown WT, Laird CD. Epigenetic variation illustrated by DNA methylation patterns of the fragile-X gene FMR1. Hum Mol Genet 1997; 6: 1791 1801.

28. Uchida T, Ohashi H, Aoki E, Nakahara $Y$, Hotta T, Murate T, Saito H, Kinoshita T Clonality analysis by methylation-specific PCR for the human androgen-receptor gene (HUMARA-MSP). Leukemia 2000; 14: 207-212.

29. Hoffman P, Milliken D, Gregg L, Davis R, Gregg J. Molecular characterization of uterine fibroids and its implication for underlying mechanisms of pathogenesis. Fertil Steril 2004; 82: 639-649.

30. Skubitz K, Skubitz A. Differential gene expression in uterine leiomyoma. J Lab Clin Med 2003; 141: 297-308.

31. Heard E. Delving into the diversity of facultative heterochromatin: the epigenetics of the inactive X chromosome. Curr Opin Genet Dev 2005; 15: 482-489.

32. Oda M, Yamagiwa A, Yamamoto S, Nakayama T, Tsumura A, Sasaki H, Nakao K, Li E, Okano M. DNA methylation regulates long-range gene silencing of an X-linked homeobox gene cluster in a lineage-specific manner. Genes Dev 2006; 20: 3382-3394.

33. Ikegami K, Ohgane J, Tanaka S, Yagi S, Shiota K. Interplay between DNA methylation, histone modification and chromatin remodeling in stem cells and during development. Int J Dev Biol 2009; 53: 203-214.

34. Ballestar E, Esteller M. The impact of chromatin in human cancer: linking DNA methylation to gene silencing. Carcinogenesis 2002; 23: 1103-1109.

35. El-Osta A. The rise and fall of genomic methylation in cancer. Leukemia 2004; 18 : 233 237.

36. Feinberg AP, Cui H, Ohlsson R. DNA methylation and genomic imprinting: insights from cancer into epigenetic mechanisms. Semin Cancer Biol 2002; 12: 389-398.

37. Iwase H, Omoto Y, Iwata H, Toyama T, Hara Y, Ando Y, Ito Y, Fujii Y, Kobayashi S DNA methylation analysis at distal and proximal promoter regions of the oestrogen receptor gene in breast cancers. Br J Cancer 1999; 80: 1982-1986.

38. Lapidus RG, Ferguson AT, Ottaviano YL, Parl FF, Smith HS, Weitzman SA, Baylin SB, Issa JP, Davidson NE. Methylation of estrogen and progesterone receptor gene 5 $\mathrm{CpG}$ islands correlates with lack of estrogen and progesterone receptor gene expression in breast tumors. Clin Cancer Res 1996; 2: 805-810.

39. Miladi-Abdennadher I, Abdelmaksoud-Damak R, Ayadi L, Khabir A, Frikha F, Kallel L, Amouri A, Frikha M, Sellami-Boudawara T, Gargouri A, Mokdad-Gargouri R. Hypermethylation of RARbeta2 correlates with high COX-2 expression and poor prognosis in patients with colorectal carcinoma. Tumour Biol 2010.

40. Wang XH, Zhang LH, Zhong XY, Xing XF, Liu YQ, Niu ZJ, Peng Y, Du H, Zhang GG, Hu Y. S100A6 Overexpression is associated with poor prognosis and is epigenetically up-regulated in gastric cancer. Am J Pathol 2010; 177: 586-597.

41. Zhang Y, Miao Y, Yi J, Wang R, Chen $\mathbf{L}$. Frequent epigenetic inactivation of deleted in lung and esophageal cancer 1 gene by promoter methylation in non-small-cell lung cancer. Clin Lung Cancer 2010; 11: 264-270.

42. Breault JE, Shiina H, Igawa M, Ribeiro-Filho LA, Deguchi M, Enokida H, Urakam S, Terashima M, Nakagawa M, Kane CJ, Carroll PR, Dahiya R. Methylation of the gamma-catenin gene is associated with poor prognosis of renal cell carcinoma. Clin Cancer Res 2005; 11: 557-564. 